\title{
Research On Fault Diagnosis Of Wind Turbine Gearbox Based On IFA-ELM
}

\author{
Shaomin Zhang ${ }^{\mathrm{a}}$, Jia Wei ${ }^{\mathrm{b}}$, Baoyi Wang ${ }^{\mathrm{c}}$ \\ ${ }^{1}$ School of Control and Computer Engineering, North China Electric Power \\ University,Baoding,071003,China \\ aemail:zhangshaomin@126.com,bemail:593414296@qq.com,cemail:wangbaoyi@126.com
}

Keyword: wind turbine; fault diagnosis; extreme learning machine; firefly algorithm

\begin{abstract}
In order to effectively improve the accuracy of fault diagnosis of wind turbine gearbox, a fault diagnosis model based on improved firefly algorithm for extreme learning machine is proposed in this paper. The extreme learning machine overcomes the shortcomings of traditional neural network, such as slow convergence rate and easy to fall into local minimum points, however, the weights and thresholds of the input layer and the hidden layer are generated in a random way, this can lead to excessive number of nodes in the hidden layer, resulting in over fitting in the training process. In view of this problem, the firefly algorithm with high searching speed and high efficiency is used to optimize the parameters of the extreme learning machine. However, because of the fixed step size, the firefly algorithm is easy to fall into the local optimum in the early stage and slows down in the late convergence. Therefore, the step size of the firefly algorithm is improved to make it change with the change of the objective function in the search process so as to improve the performance of the firefly algorithm. The experimental results show that compared to the standard ELM, GA-ELM, and FA-ELM networks, the improved firefly algorithm for extreme learning machine that proposed in this paper has a higher prediction accuracy.
\end{abstract}

\section{Introduction}

Gearbox is the key component of the wind turbine, its normal operation is related to the working performance of the whole unit. Due to the complex structure and the poor working environment, the gearbox is very easy to malfunction ${ }^{[1]}$. According to statistics, the fault of gearbox accounts for about $60 \%{ }^{[2]}$ of the total fault of wind turbine. Due to the gearbox space is narrow and located in the upper air, it is very difficult to maintain. Therefore, it is important to diagnose the fault of gearbox quickly and accurately, which is of great significance to reduce the cost of wind power operation and maintenance and the operation reliability of wind turbines.

In recent years, many scholars have done a lot of research on fault diagnosis of gear box. In this paper the extreme learning machine with faster training speed and better generalization ability is applied to fault diagnosis of gearbox of wind turbine. The Extreme Learning Machine(ELM)is a new learning algorithm based on single hidden layer feedforward neural network proposed by Huang guangbin in $2004^{[3]}$. Different from the traditional neural network, the most important feature of ELM algorithm is the input weights and hidden layer bias are generated during the training process randomly. The whole process is completed at once, without iteration. Compared with the traditional neural network, ELM algorithm is faster, and the generalization ability of algorithm is stronger ${ }^{[4-7]}$. At present, the ELM algorithm has been applied in the fields of face recognition $^{[8]}$, image classification ${ }^{[9]}$, fault diagnosis ${ }^{[10]}$ and load forecasting ${ }^{[1,12]}$.

However, the input parameters of the input layer and the threshold of the hidden layer are generated randomly, which affect the stability of the network structure to a certain extent. Whether the parameters selection are proper or not affect the performance of the ELM algorithm $^{[13]}$. In order to obtain better weights and bias, the meta heuristic optimization algorithm - firefly algorithm is used to optimize the ELM algorithm in this paper. 


\section{Extreme Learning Machine Algorithm}

The mathematical expression of extreme learning algorithm is as follow:

$$
y_{j}=\sum_{i=1}^{l} \beta_{i} g_{i}\left(x_{j}\right)=\sum_{i=1}^{l} \beta_{i} g_{i}\left(\omega_{i} \cdot x+b_{i}\right), \quad j=1,2,3, \cdots, N
$$

In formula (1), $w_{i}=\left[w_{i 1}, w_{i 2}, \cdots, w_{i n}\right]$ is the connection weight of the ith node between the input layer and the hidden layer, $\beta_{i}=\left[\beta_{i 1}, \beta_{i 2}, \cdots, \beta_{i n}\right]^{\mathrm{T}}$ is the connection weight of the ith node between the hidden layer and the output layer, $x_{i}=\left[x_{i 1}, x_{i 2}, \cdots, x_{i n}\right]$ is the input value, $g\left(x_{i}\right)$ is the excitation function of the hidden layer, $y_{j}=\left[y_{j 1}, y_{j 2}, \ldots, y_{j m}\right]$ is the output value of the neural network.

The formula (1) can be abbreviated as:

$$
H \beta=Y
$$

The connection weights between the hidden layer and the output layer can be obtained by solving the least square solution of the linear equations:

$$
\|H \beta-T\|=\left\|H H^{+} T-T\right\|=\min _{\beta}\|H \beta-T\|
$$

The least square solution is:

$$
\hat{\beta}=H^{+} T
$$

Among them, $H^{+}$is the Moore-Penrose generalized inverse matrix of $H$.

\section{Firefly Algorithm}

The firefly algorithm is described as follows:

Generate $\mathrm{m}$ random fireflies initially $X=\left(X_{1}, X_{2}, \cdots, X_{m}\right)$. The i-th firefly represents the firefly's position in the D-dimensional search space and is also a potential solution, and then finds the optimal solution iteratively. The brightness of the firefly indicates the position of the quality of the position, and determine the direction of the movement of the firefly, the higher the brightness indicates that the location is better, the better the value of the objective function.

In each iteration, fireflies are attracted to each other to update the position, If the brightness of firefly $j$ is less than the brightness of firefly $i$, firefly $i$ remains stationary and firefly $j$ moves toward firefly $i$ :

$$
X_{j}^{k+1}=X_{j}^{k}+\beta_{i j}\left(X_{i}^{k}-X_{j}^{k}\right)+\alpha\left(\text { rand }-\frac{1}{2}\right)
$$

In formula (5): $i, j=1,2, \cdots, m, k$ is the current iteration number; $\beta_{i j}$ is the relative attractiveness of firefly $i$ and firefly $j . \alpha$ is the step factor, and is a constant, usually $\alpha \in[0,1]$; rand is evenly distributed on the random number[0,1].Compared with the genetic algorithm and particle swarm algorithm, the firefly algorithm has no mutation and cross operation, so it is easier to implement and operate, and the convergence speed is faster. The optimal algorithm of FA, GA, and PSO is shown in Table 1, where the population size is 100 , the number of iterations is 50 , the light absorption coefficient of FA algorithm is 1 , and the maximum attractive force is 1 . PSO algorithm learning factor $C_{1}=C_{2}=2$, and the crossover probability and mutation probability of GA are both 0.5 .

Table 1 Training time of each algorithm (unit: s)

\begin{tabular}{cccc}
\hline function & GA & PSO & FA \\
\hline$f_{1}(x)$ & 23.8564 & 18.6575 & 12.8529 \\
\hline$f_{2}(x)$ & 25.8736 & 20.6436 & 10.3795 \\
\hline$f_{3}(x)$ & 24.2561 & 19.7574 & 14.6538 \\
\hline
\end{tabular}

In Table 1, the three functions are:

(1) Schaffer F6 


$$
f_{1}(x)=\frac{\sin ^{2} \sqrt{x_{1}^{2}+x_{2}^{2}}-0.5}{\left[1+0.001\left(x_{1}^{2}+x_{2}^{2}\right)\right]^{2}}-0.5, x_{i} \in[-100,100]
$$

(2) Generalized Rastrigin

$$
f_{2}(x)=\sum_{i=1}^{d}\left[x_{i}^{2}-10 \cos \left(2 \pi x_{i}\right)+10\right] x_{i} \in[-5.12,5.12]
$$

(3) Ackley

$$
f_{3}(x)=-20 \exp \left(-0.2 \sqrt{\frac{1}{d} \sum_{i=1}^{d} x_{i}^{2}}\right)-\exp \left(\frac{1}{d} \sum_{i=1}^{d} \cos \left(2 \pi x_{i}\right)\right)+20+e, x_{i} \in[-32,32]
$$

It can be seen from Table 1 that when the convergence to a given precision, compared to the genetic algorithm and particle swarm algorithm, the running time of firefly algorithm is shorter, so the firefly algorithm is selected to optimize the extreme learning machine algorithm.

But the firefly algorithm itself is easy to be trapped in the local optimal and converge slow in later stage. In the algorithm, the step size is kept constant, and the algorithm is easy to "premature" and converges to the local extremum. the algorithm can not converge even if the parameters are set improperly. In order to improve the performance of the algorithm, the FA algorithm is improved in this paper.

\section{Improved firefly algorithm (IFA)}

New calculation method of relative attractiveness. In the standard firefly algorithm, the relative attractiveness is calculated as follows:

$$
\beta_{i j}=\beta_{0} e^{-r_{i j}^{2}}
$$

As can be seen from formula (6), the relative attractiveness is only related to the maximum attraction and the relative distance. Moreover, the maximum degree of attraction is usually taken as a constant 1, which corresponds to the calculation of relative attractiveness only taking into account the relative distance between fireflies.

However, in fact, the magnitude of the relative attractiveness of the two fireflies is not only related to their relative distance, but also to the relative brightness of the two fireflies, which in turn are closely related to the objective function value. Therefore, the relative attractiveness of the firefly is also closely related to the objective function value. The improved relative attractiveness is calculated as follows:

$$
\beta_{i j}=I_{i j} e^{-r_{i j}^{2}}
$$

In formula (7), $I_{i j}$ is the relative brightness between firefly $i$ and firefly $j, I_{i j}=\left|I_{i}-I_{j}\right|, \gamma$ is the light intensity absorption coefficient, $\gamma \in[0.01,100]$,and $r_{i j}$ is the distance between firefly $i$ and firefly $j$.

Adaptive variable step size calculation method. The most important parameter that affects the movement of the firefly to the optimum position is the step factor $\alpha$, but in the standard firefly algorithm it is a constant and is fixed. In the latter part of the algorithm, the firefly has moved to the local or global extreme point, if the step is too large, it will cause the firefly to oscillate repeatedly near the extremum point, affecting the local search ability of the algorithm; In the early stage of the algorithm, if the step size is too small, it is unfavorable for the firefly to converge to the extreme point rapidly, which affects the global searching ability and the convergence speed of the algorithm. Therefore, the fixed step can not satisfy both the initial and the late requirements of the algorithm. The fixed step is the main reason for the problem of slow convergence, low precision, and local optimality. Aiming at the above problems, an adaptive variable step size firefly algorithm based on the objective function is proposed in this paper. The step size calculation method is as follows: 


$$
\left\{\begin{array}{c}
\lambda(k)=\frac{1}{m} \sum_{i=1}^{m}\left(f\left(X_{i}(k)\right)-f\left(X_{\text {best }}(k)\right)\right) \\
\alpha(k)=e^{-\frac{\lambda(k(k))}{\lambda(k)}}
\end{array}\right.
$$

In formula (8), $f\left(X_{i}(k)\right)$ denotes the objective function value corresponding to the $i$ th fireflies of the $k$ th iteration, and $f\left(X_{\text {best }}(k)\right)$ denotes the objective function value corresponding to the optimal firefly of the $k$ th iteration.

\section{Improved Firefly - Extreme Learning Algorithm}

The basic idea of the improved firefly algorithm to optimize the extreme learning machine is: The network structure of the ELM is determined by the input and output fault types of wind turbine gearbox. In the FA algorithm, the firefly individual is encoded as the input layer and the hidden layer bias of the ELM . The fitness function $f\left(X_{i}\right)$ is the mean square error $R M S E$ between the actual output and the expected output of the ELM. The brightness of the firefly is $I_{i}=f\left(X_{i}\right)$. The firefly individual attracts each other through the brightness and the relative brightness, it will reach the global optimum point after location update constantly. The optimal firefly is assigned to the input weight and bias and then the hidden layer output matrix and output weight are calculated to obtain the optimal algorithm based on the improved firefly algorithm. And then this model can be used to diagnose the fault of gearbox of wind turbine. The flow chart of fault diagnosis of IFA-ELM algorithm is shown in Fig.1.

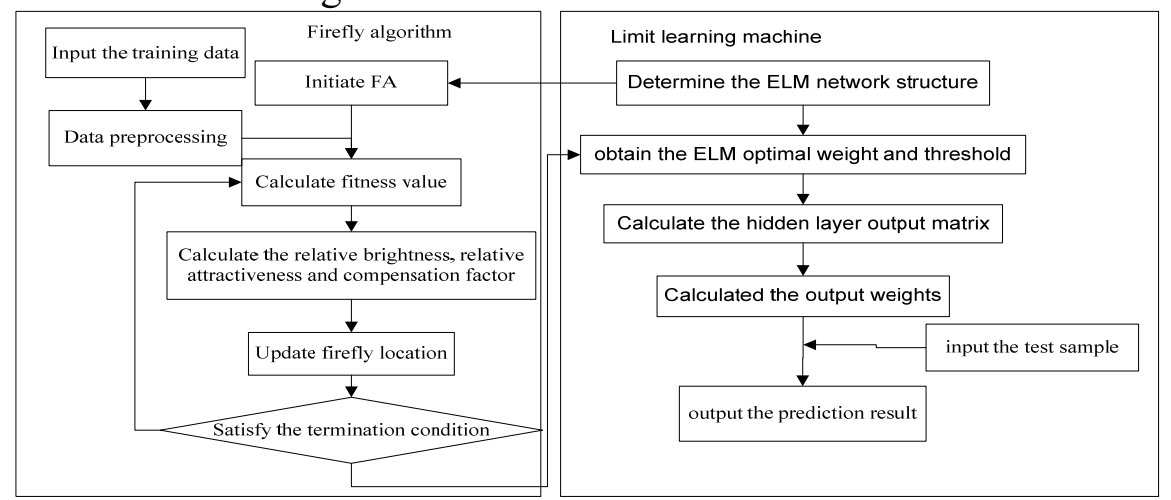

Fig.1 The flow chart of fault diagnosis of IFA-ELM algorithm

The model consists of three parts: ELM neural network structure determination, firefly algorithm optimization and ELM neural network training and prediction. The specific steps are as follows:

(1) Determine the network structure of the ELM. The input and output layer nodes of ELM neural network are determined according to the input and output of fault type of gearbox of wind turbine. The number of hidden layer nodes is set manually.

(2) Initialize the firefly population. Randomly generate $\mathrm{m}$ fireflies and encode them. The real string consists of two parts: the input layer weight and hidden layer bias. Each individual contains the weights and bias of ELM algorithm. And set the light intensity absorption coefficient $\gamma$ and the maximum number of iterations $t$.

(3) Calculate the fitness function value. The elements of the firefly's position vector are decoded, and the decoded value is the weight and bias of the ELM and assigned it to the ELM network. The ELM neural network is trained by using the training samples. The mean square error $R S M E$ of the actual output and expected output is taken as the fitness function of each firefly, where $\mathrm{N}$ is the number of nodes in the output layer of the ELM network.

(4) Update the firefly location. Calculate the brightness of each firefly individual to determine the direction of the firefly movement. Calculate the relative attractiveness of fireflies according to formula(3).And calculate the step size according to formula(4).And update the position of firefly according to formula(1). 
(5) If the mean square error $A$ is lower than the set precision or the maximum number of iterations is reached, then go to step (6); Otherwise go to step (3);

(6) The position of the optimal fireflies is assigned to the weights and bias of the ELM algorithm to calculate the hidden layer output matrix $H$, and then the Moore-Penrose generalized inverse matrix of $H$ is calculated too.

(7) Calculate the output layer weight.

(8) Finish the training. Input the test sample and get the test results.

\section{Experiment and Result Analysis}

The data set used in this paper is the actual operation data of a wind turbine gearbox for a wind farm. The fault data and normal data of the gearbox are selected from the collected data set, which the training set and the test set are randomly divided by 3: 2 to verify the accuracy of the IFA-ELM algorithm proposed in this paper. Table 3 shows the three most common fault types and one normal condition for the gearbox used in the experiment.

Table 2 Fault type of gearbox

\begin{tabular}{c|c} 
sequence number & fault type \\
1 & normal \\
2 & gear spot corrosion \\
3 & gear wear \\
4 & gear tooth broken \\
\hline
\end{tabular}

In the IFA-ELM algorithm, the size of the firefly population is 30, the iteration number is 300 , the light absorption coefficient is 1, the fitness function is the ELM training error mean square error, and the ELM hidden layer activation function is sigmoid function.

The process of parameters optimization of ELM optimized by FA and IFA are shown in Figure 3.It can be seen from the figure that FA algorithm finds the optimal solution in the 162nd generation, and IFA algorithm finds the optimal solution in the 84th generation.IFA algorithm finishes the optimization more quickly than the FA, and improves the efficiency of the fault diagnosis.

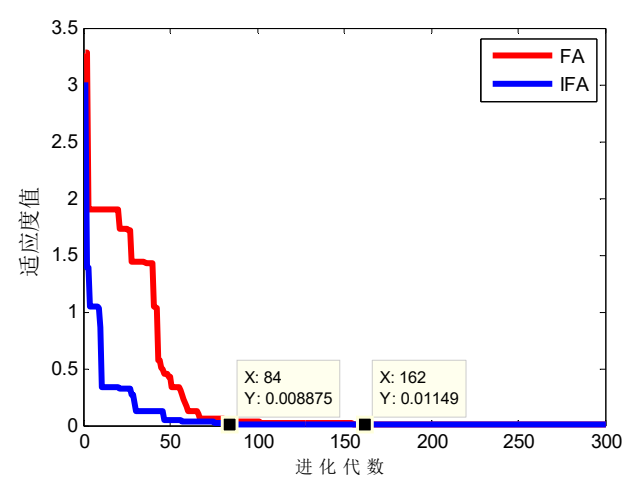

Fig.2 Comparison of optimization performance between IFA and FA

The training datasets were input to the ELM, GA-ELM, FA-ELM and IFA-ELM networks, and the test datasets were predicted. Ten experiments were carried out, taking the average. The prediction accuracy of the four models is shown in Table 4.As can be seen from Table 4, compared with ELM, GA-ELM, FA-ELM neural network, IFA-ELM network has higher correct rate and better prediction effect, which proves the effectiveness of the algorithm.

Table 3 prediction accuracy of different models

\begin{tabular}{c|c|c|c|c}
\hline fault type & ELM & GA-ELM & FA-ELM & IFA-ELM \\
normal & 91.37 & 96.69 & 97.48 & 100 \\
Gear spot corrosion & 89.8 & 95.08 & 95.44 & 98.55 \\
gear wear & 86.95 & 96.17 & 96.10 & 99.15 \\
gear tooth broken & 90.41 & 95.43 & 94.95 & 98.85 \\
\hline
\end{tabular}




\section{Conclusion}

According to the requirement of the effectiveness and fastness of fault diagnosis of gearbox of wind turbine, an improved firefly algorithm - extreme learning machine algorithm is proposed in this paper. Compared with ELM, FA-ELM and FA-ELM, the proposed IFA-ELM algorithm can greatly improve the prediction accuracy and meet the requirement of fault diagnosis of gearbox of wind turbine.

\section{Acknowledgement}

In this paper, the research was sponsored by the National Natural Science Foundation of China(Project No.)and Scientific Research Project of Hebei Province(Project No.).

\section{References}

[1] Li Junfeng,Cai Fengbo, Qiao Liming. Report on development of wind power in China [R].China Resources Comprehensive Utilization Association Renewable Energy Professional Committee.2012.

[2] Global Wind Energy Council .Global Wind Report Annual Market Update 2012 [R].2013.

[3] Guang-Bin Huang, Qin-Yu Zhu, Chee-Kheong Siew. Extreme learning machine: a new learning scheme of feedforward neural networks[C]. //2004 IEEE International Joint Conference on Neural Networks. Budapest, Hungary: IEEE, 2004:985-990.

[4] Huang G B, Ding X, Zhou H. Optimization method based extreme learning machine for classification[J]. Neurocomputing, 2010, 74( 1):155-163.

[5] Huang G B,Zhou H, Ding X, Zhang R. Extreme Learning Machine for Regression and Multiclass Classification[J]. IEEE Transactions on Systems, Man and Cybernetics, Part B (Cybernetics),2012,42(2):513-29.

[6] Cambria Erik, Huang Guang-Bin,et al. Extreme learning machines[J]. IEEE Intelligent Systems,2013,28(6):30-59.

[7] Ma Chao.Research on classification algorithm and its application based on meta heuristic optimization for extreme learning machine[D].Jilin University.2014.

[8] Zong, W. and G.-B. Huang. Face recognition based on extreme learning machine[J]. Neurocomputing, 2011, 74(16):2541-2551.

[9] Li Xiaodong.The theory and algorithm of nuclear extreme learning machine and its application in image processing[D].Zhejiang University.2014.

[10] Gao Fei,Li Hongru,Xu Baohua.Application of extreme learning machine optimized by ICPSO in fault diagnosis[J].China Mechanical Engineering.2013,38020:2753-2757.

[11] Wang Baoyi,Zhao Shuo,Zhang Shaomin.Distributed power load forecasting algorithm based on cloud computing and extreme learning machine[J]. Power System Technology.2014,02:526-531.

[12] Mao Li,Wang Yuntao,Liu Xingyang,Li Zhaofeng.Short term electric power load forecasting method based on Improved Extreme Learning Machine[J].Power System Protection and Control.2012,20:140-144.

[13] Wang, Y., F. Cao, and Y. Yuan. A study on effectiveness of extreme learning machine[J]. Neurocomputing, 2011, 74(16): 2483-2490. 